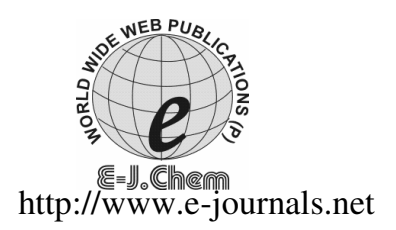

ISSN: 0973-4945; CODEN ECJHAO

E-Journal of Chemistry

2011, 8(2), 703-710

\title{
Semi-Empirical Predictions on the Structure and Properties of ent-Kaurenoic Acid and Derivatives
}

\author{
JOSE ISAGANI B. JANAIRO ${ }^{\S *}$, GERARDO C. JANAIRO ${ }^{\S}$ \\ and DERRICK ETHELBHERT C. YU \\ ${ }^{\S}$ Materials Science and Nanotechnology Unit \\ Center for Natural Sciences and Environmental Research \\ De La Salle University, 2401 Taft Avenue, Manila, Philippines \\ *Department of Physics, De La Salle University \\ 2401 Taft Avenue, Manila, Philippines \\ Department of Chemistry, De La Salle University \\ 2401 Taft Avenue, Manila, Philippines \\ jose.isagani.janairo@dlsu.edu.ph
}

Received 25 July 2010; Accepted 2 October 2010

\begin{abstract}
The physicochemical properties of ent- kaurenoic acid model derivatives, which possibly influence its therapeutic application, were calculated. Results revealed that the molecule possess favourable attributes which renders it possible to be considered as a drug lead only that its very hydrophobic nature can result to poor bioavailabilty, low absorption and poor systemic circulation. In silico simulations revealed that this setback can be overcome by introduction of hydroxyl group to the tertiary carbon of entkaurenoic acid employing $m$-CPBA catalyzed hydroxylation, thus, unleashing its full drug potency. Moreover, molecular similarity analyses derived from semi-empirical calculations between ent-kaurenoic acid and a set of kaurane diterpenoids showed differences in hydrophobic complementarity, size and electronic properties despite possessing nearly identical molecular frameworks, thus, arriving in a generalization for their observed mechanistic differences on acting on different targets.
\end{abstract}

Keywords: Semi-empirical calculations, Molecular similarity analysis, Ent-kaurenoic acid

\section{Introduction}

Natural products have played a significant role in pharmaceutical research since plantderived molecules have provided an arsenal of biologically active compounds possessing diverse activity such as anti-tumour, antibacterial, anti-inflammatory, etc ${ }^{1-2}$ which served as 
lead candidates and have been then developed into useful drugs. One particularly interesting natural product is ent-kaurenoic acid which is known to exhibit promising activities such as cytotoxicity $^{3}$, smooth muscle relaxant ${ }^{4}$, antibacterial ${ }^{5}$, hypoglycaemic ${ }^{6}$, etc.<smiles>C=C1CC2CC3C4CCCC(C)(C)C4CC(O)C3C(C(=O)O)C2C1</smiles>

1

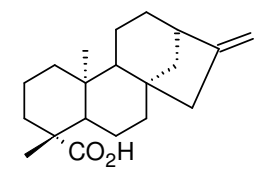

ent-kaurenoic acid (L)

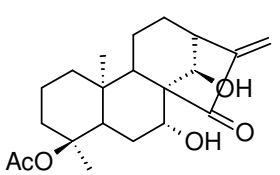

4

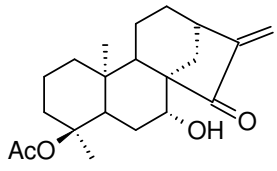

2

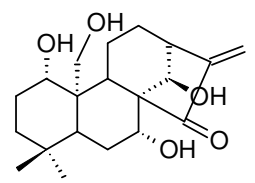

5

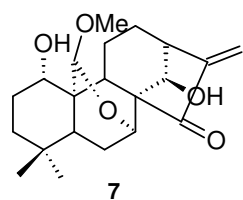

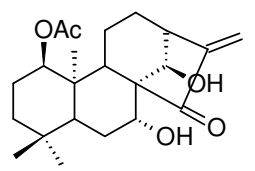

3

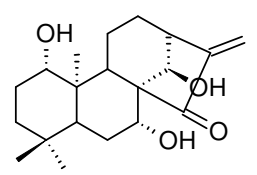

6

Figure 1. ent-kaurenoic acid and kaurane diterpenoids

The observed glucose lowering effect of ent-kaurenoic acid is perhaps attributed to its ability to inhibit NF- $\mathrm{B}$ (nuclear factor $-\kappa \mathrm{B}$ ) which, once activated is responsible for the transcription of inflammatory mediators that have been established to contribute to the onset of insulin resistance ${ }^{7}$ NF- $\mathrm{kB}$ is a transcription factor which is usually bound to its inhibitory partner I $\kappa \mathrm{B}$ thereby rendering it inactive. Upon stimulus activation, I $\kappa \mathrm{B}$ is phosphorylated by IKK resulting to its degradation; NF- $\mathrm{KB}$ is thus liberated and translocated into the nucleus in which it shall facilitate the transcription of several genes involved in inflammatory signalling process ${ }^{8}$. The NF- $\mathrm{KB}$ inhibitory effect of ent-kaurenoic acid is exerted through covalent modification of IKK by a nucleophilic addition with the cysteine residues of the kinase $^{9}$. It is interesting to note that kaurane diterpenoids, compounds that are analogous with ent-kaurenoic acid also possess NF- $\mathrm{BB}$ inhibitory activity only that they covalently modify the DNA binding site of NF- $\mathrm{KB}$ in a manner similar to that of ent-kaurenoic acid ${ }^{10-11}$.

Recognizing the potential of ent-kaurenoic acid, the study presented herein aims to evaluate its physico chemical properties through theoretical assessment of its structure which shall provide invaluable insight for lead optimization studies and synthetic derivatization of the molecule. Molecular comparison shall also be conducted in order to probe the similarities and differences existing between ent-kaurenoic acid and the aforementioned kaurane diterpenoids which might explain the difference in their preferred targets despite sharing a common structural framework. The data obtained will supply additional knowledge on the binding behaviour of the ligands to their respective targets since site directed mutagenesis, which only determines the significant amino acid residue that participates in the inhibition process, was the predominant method utilized by other researchers when mechanistic studies were performed ${ }^{11}$.

\section{Methodology}

SPARTAN 08 V.1.2.0 (Wavefun, Inc.) was used for all calculations performed in this research. The lowest energy conformer of the molecule was identified using a Monte Carlo 
algorithm through a stepwise multiple rotations of the molecules' single bonds. The generated lowest energy conformer was then subjected to geometry optimization using AM1 semi-empirical quantum calculations from which their corresponding energy profile was calculated. Chemical descriptors such as structural alignment, HOMO and LUMO energies, index of intrinsic reactivity, $\log$ P, PSA and nucleophilic susceptibility were calculated as well.

\section{Results and Discussion}

\section{Evaluation of properties}

The physicochemical properties of ent-kaurenoic acid which possibly influences its known biological activities were evaluated against the extended Lipinski's rule that were outlined along with several added parameters presented by Young ${ }^{12}$.

Table 1. Tabulated Properties of ent-Kaurenoic acid

\begin{tabular}{ccc}
\hline Property & $\begin{array}{c}\text { ent- Kaurenoic acid } \\
\text { (calculated) }\end{array}$ & Ideal value \\
\hline Hydrogen bond donors & 1 & $\leq 5$ \\
Hydrogen bond acceptors & 1 & $\leq 10$ \\
Molecular weight & 302.46 & $\leq 500$ \\
$\log P$ & 6.37 & $0-4$ \\
PSA & 31.603 & $<140$ \\
Number of rotatable bonds & 4 & $\leq 5$ \\
\hline
\end{tabular}

The formation of hydrogen bonds is an integral part of the molecular recognition process since such non-covalent interactions provide the basis of molecular specificity among receptor-ligand processes ${ }^{13}$. However, excessive number of hydrogen bond donors and acceptors result to poor permeability of the molecule across the lipid bilayer ${ }^{14}$; hence a restriction has been introduced to serve as a guide for high throughput screening. Likewise, the molecular weight, $\log$ P and PSA (polar surface area) also serve as a descriptor of bioavailabilty and absorption of the molecule into the cells. $\log \mathrm{P}$ is the partition coefficient of a compound in an octanol / water mixture ${ }^{15}$ wherein $\log \mathrm{P}$ describes the general hydrophobicity character of the compound. A $\log \mathrm{P}$ value exceeding the suggested ideal value is indicative of an overall hydrophobic character which will provide the molecule with low solubility to water, hence poor systemic circulation. On the other hand, PSA predicts the overall polarity of the molecule by taking into account the different atoms attached to it; PSA calculations have been used as a descriptor for passive epithelial permeability ${ }^{16}$ and serves as the dominant descriptor of oral bioavailability and brain penetration ${ }^{17}$. If the molecule is known to occupy the active site of an enzyme or a cavity of a biomolecule, then the number of rotatable bonds must be considered in order to understand the thermodynamics associated with the process. If the degrees of freedom conferred upon by the rotatable bonds of the molecule are great, an energy penalty is introduced to the system in order for the molecule to achieve its lowest energy conformer, thus making the binding process less favourable ${ }^{12}$.

Analysis of the results indicates that ent-kaurenoic acid has great potential as a drug lead since its physicochemical properties coincides with the ideal values associated with each parameter; although the very hydrophobic nature is a potential hindrance since it can lead to poor bioavailabilty, low absorption and poor systemic circulation. Therefore, incorporation of polar functionalities can be a strategy to improve the bioavailability and solubility of ent-kaurenoic acid but the favourable attributes of compound must not be sacrificed entirely. 


\section{Molecular comparison of ent-kaurenoic acid with kaurane diterpenoids}

The tetracyclic framework is a shared structural framework common between ent-kaurenoic acid and kaurane diterpenoids. Since the term "similarity" is vague and ambiguous if used in a qualitative context, a quantitative description is herein provided. The conformational analysis of ent-kaurenoic acid resulted to the generation of 9 conformers, in which the lowest energy conformer has a population density of 52.32\%. Kaurane diterpenoids were also subjected to the same procedure and their corresponding population density ranges from 24.09 to $96.43 \%$. After geometry optimization, the kaurane diterpenoids were aligned with ent-kaurenoic acid and an alignment score is thereafter produced.

Table 2. Alignment scores of compounds 1-7 with ent-kaurenoic acid

\begin{tabular}{cc}
\hline Compound & Score \\
\hline 1 & 0.97 \\
2 & 0.93 \\
3 & 0.92 \\
4 & 0.93 \\
5 & 0.92 \\
6 & 0.92 \\
7 & 0.88 \\
\hline
\end{tabular}

As expected, the compounds exhibited a high degree of structural similarity with entkaurenoic acid with 0.88 as the lowest alignment score. The observed high degree of structural similarity existing among the molecules suggests that such a structural motif meets the steric complementarities of the binding site of both targets which is a requirement for host-ligand recognition ${ }^{18}$.

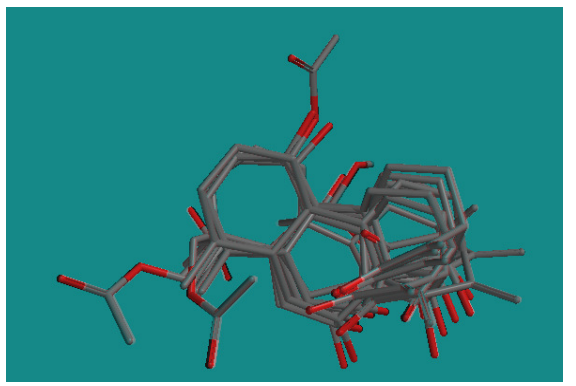

Figure 2. Structural alignment of the 7 compounds with ent-kaurenoic acid

A plot of volume vs. area indicates that ent-kaurenoic acid is relatively smaller and occupies lesser space compared to the kaurane diterpenoids. This can be a possible point of differentiation since the nucleophilic cysteine residue in which ent-kaurenoic acid forms an adduct may not be able to attack the other larger molecules since they cannot adequately fit the cavity.

As previously discussed, the lack of hydroxyl functionality of ent-kaurenoic acid has resulted to its very hydrophobic nature which sets it apart from the kaurane diterpenoids as seen from the two-descriptor model wherein ent-kaurenoic acid is dispersed from the group. The hydrophobic discrepancy between ent-kaurenoic acid and kaurane diterpenoids affords another point of differentiation since hydrophobic complementarity is another prerequisite for molecular recognition ${ }^{18}$. Therefore, ent-kaurenoic acid adopts a very different conformation from the kaurane diterpenoids in order to match the hydrophobic regions of its corresponding target. 


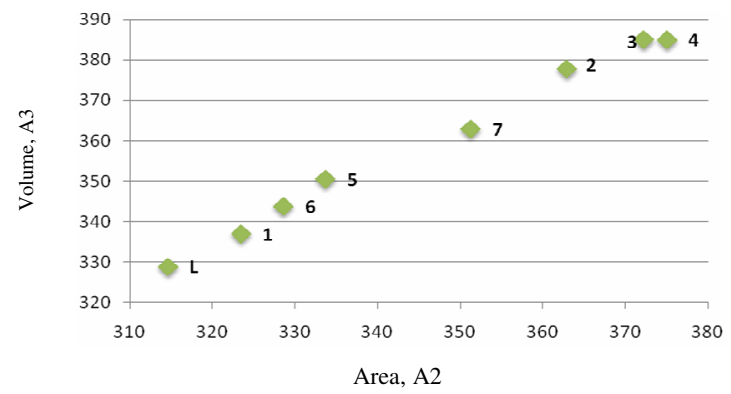

Figure 3. Correlation between area and volume

A two-descriptor model of log P and PSA was also constructed in order to compare the relative solubility of the molecules.

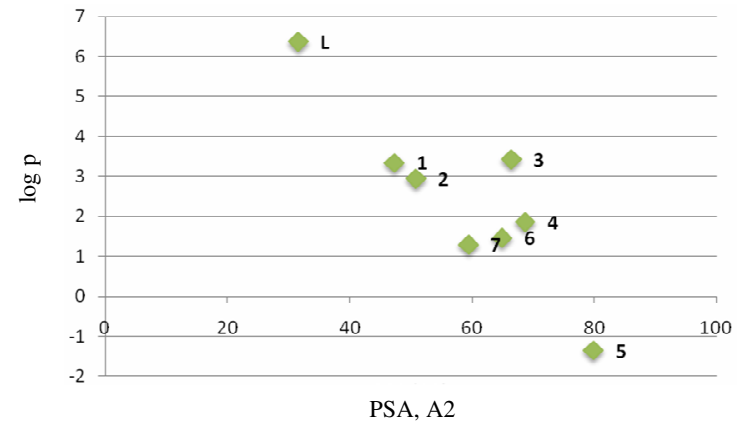

Figure 4. Two - descriptor model for solubility and absorption

As previously mentioned, the mode of NF- $\mathrm{KB}$ inhibition involves the nucleophilic addition of the sulfhydryl group of cysteine at the carbonyl carbon. Since ent-kaurenoic acid does not possess a carbonyl functionality at the bridgehead position, it is logical to assume that the carbonyl group of the carboxylic acid will be the subject of the nucleophilic attack. Calculated LUMO density maps of the molecules indicated that the carbonyl group at the bridgehead position and the carboxylic acid moiety (blue region) are indeed the groups most susceptible to nucleophilic attack for kaurane diterpenoids and ent-kaurenoic acid, respectively.

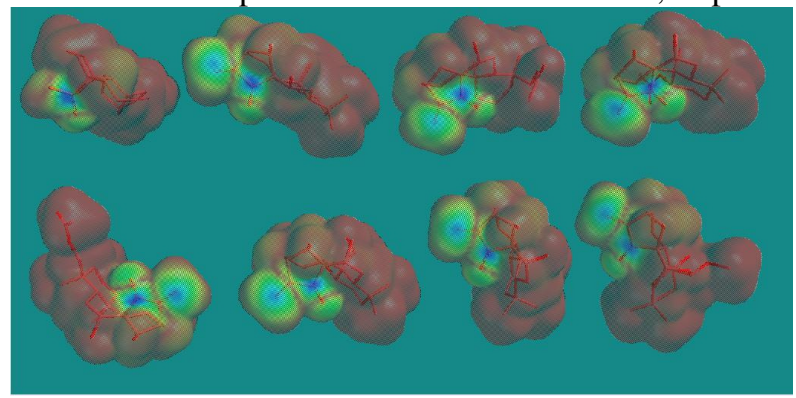

Figure 5. LUMO density map (from L-R: L, 1,2,3,4,5,6,7)

The calculated HOMO and LUMO energies revealed, as testified by the relatively smaller magnitude of the $\mathrm{E}_{\mathrm{LUMO}}$, that the molecules exhibit more electrophilic and electron accepting properties than their corresponding counterparts ${ }^{19}$. It is also noteworthy to point out that ent-kaurenoic acid possessed the greatest value of $\eta$ or index of intrinsic reactivity which indicates that ent-kaurenoic acid is the least reactive among the compounds. 
Table 3. HOMO and LUMO Energies

\begin{tabular}{cccc}
\hline Compound & $\mathrm{E}_{\text {номо, }} \mathrm{eV}$ & $\mathrm{E}_{\text {LUMO, }} \mathrm{eV}$ & $\begin{array}{c}\eta=\mathrm{E}_{\text {LUMO }} \mathrm{E} \\
\text { Hомо }\end{array}$ \\
\hline Ent -kaurenoic acid & -9.67 & 1.18 & 5.425 \\
1 & -10.11 & -0.02 & 5.045 \\
2 & -10.31 & -0.08 & 5.115 \\
3 & -10.34 & -0.17 & 5.085 \\
4 & -10.43 & -0.34 & 5.045 \\
5 & -10.23 & -0.24 & 4.995 \\
6 & -10.23 & -0.15 & 5.04 \\
7 & -10.12 & -0.1 & 5.01 \\
\hline
\end{tabular}

Since ent-kaurenoic acid has the highest energy band gap among the group of molecules, it is considered a "hard molecule" whereas the others are considered "soft molecules" by virtue of their relatively small energy band gap. Since chemical reactions involve alterations with electron density distribution pattern, a "softer" molecule translates into greater reactivity ${ }^{20}$. Ent-kaurenoic acid also obtained the highest value of LUMO energy which can be correlated to solvation models. A high $\mathrm{E}_{\mathrm{LUMO}}$ means weaker solvation ${ }^{21}$, which is consistent with previous data highlighting the very hydrophobic nature of ent-kaurenoic acid as well as sound theoretical agreement among the different models.

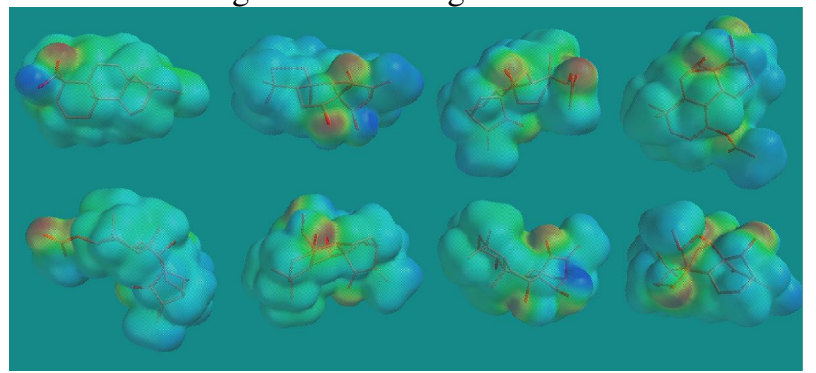

Figure 6. Potential density map (from L-R: L, 1,2,3,4,5,6,7)

The calculated density potential maps indicated that the kaurane diterpenoids possessed multiple sites where electron density is concentrated (red region), whereas ent-kaurenoic acid only had one area. Since electrostatic interactions play a crucial role in ligand - protein binding mechanism, this suggests that the electrostatic differences between ent-kaurenoic acid and the kaurane diterpenoids is also responsible for the observed selective affinity they exhibit to their respective targets. This is due to the fact that the ligand orients itself towards the binding site in such a way that the polarized centers of the ligand are aligned with their corresponding counterparts in order to facilitate optimum contact with the binding site ${ }^{19}$.

\section{Structure optimization of ent-kaurenoic acid}

The inherent hydrophobicity of ent-kaurenoic acid poses an imminent hindrance to its possible therapeutic applications. In order to improve its bioavailability and absorption, an experimentally-possible synthetic addition of a hydroxyl functionality to the ent-kaurenoic acid was theoretically simulated and the properties of the resulting ent-kaurenoic acid derivatives were examined. Using the $m$-CPBA ( $m$ - chloroperbenzoic acid) catalyzed hydroxylation as the model reaction, the three possible products were subjected to the same calculations that were performed with the other molecules. Since the reaction involves a radical substitution mechanism, the tertiary carbon centers were the point of hydroxylation ${ }^{22}$. 


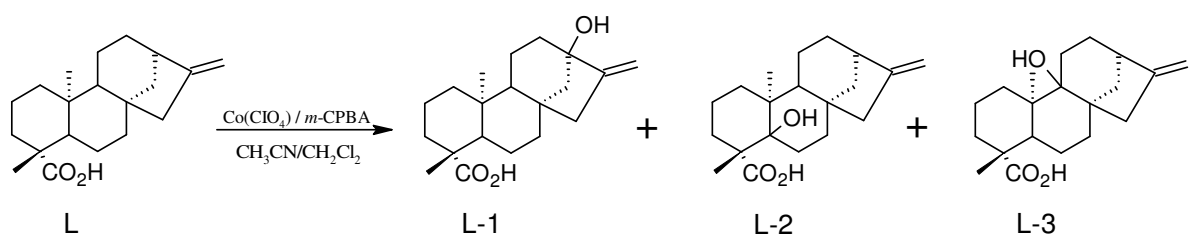

Figure 7. Proposed hydroxylation method and the corresponding products

Table 4. Comparison of properties among ent-kaurenoic acid and its hydroxylated derivatives

\begin{tabular}{lcccc}
\hline \multicolumn{1}{c}{ Property } & $\begin{array}{c}\text { Ent-Kaurenoic } \\
\text { Acid }\end{array}$ & L-1 & L-2 & L-3 \\
\hline Hydrogen bond donors & 1 & 2 & 2 & 2 \\
Hydrogen bond acceptors & 1 & 2 & 2 & 2 \\
Molecular Weight & 302.46 & 318.45 & 318.45 & 318.45 \\
$\operatorname{logP}$ & 6.37 & 4.52 & 4.44 & 4.78 \\
PSA, $\mathrm{A}^{2}$ & 31.603 & 50.90 & 45.41 & 45.96 \\
Number of rotatable bonds & 4 & 5 & 5 & 5 \\
$\mathrm{E}_{\text {HOMO }}, \mathrm{eV}$ & -9.67 & -9.87 & -9.65 & -9.68 \\
$\mathrm{E}_{\text {LUMO }}$ eV & 1.18 & 1.15 & 1.05 & 1.10 \\
$\eta=\mathrm{E}_{\text {LUMO }} \mathrm{E}_{\text {HOMO }} / 2$ & 5.425 & 5.510 & 5.350 & 5.390 \\
\hline
\end{tabular}

The resulting hydroxylated derivatives are expected to possess greater bioavailability since its hydrophobic property was significantly decreased. Other properties of the hydroxylated derivatives were still within the metrics of drug-likeness which means that the addition of a hydroxyl functionality did not diminish their drug-likeness but rather enhanced it. Furthermore, the steric and electronic properties of the hydroxylated derivatives were almost similar with that of ent-kaurenoic acid which suggests that derivatives will also exhibit the same preferential binding to the target of ent-kaurenoic acid.

\section{Conclusion}

Ent-kaurenoic acid possesses promising drug potential according to the results of drug likeness evaluation and incorporation of hydroxyl functionality further improve its drug potency mainly because ent-kaurenoic acid's very hydrophobic nature was greatly diminished. The similarity existing between ent-kaurenoic acid and its derivatives with respect to steric and electronic properties suggest that the derivatives will also exert its activity in a similar mechanism.

Results of molecular comparison of ent-kaurenoic acid with kaurane diterpenoids revealed that the two classes of compounds share a common structural framework but differ in size, hydrophobicity and electronic properties which are essential for ligand-receptor recognition. Ent - kaurenoic acid was deemed to be the least reactive and most hydrophobic among the group of molecules and this might explain the observed target difference. It can be postulated that since the kaurane diterpenoids are much bigger relative to ent-kaurenoic acid, their inability to inhibit IKK might arise from the idea that they cannot fit into the active site of the enzyme. On the other hand, the less reactive nature of ent-kaurenoic acid might explain its inability to inhibit the DNA binding domain of NF- $\mathrm{KB}$ since the nucelophilic sulfhydryl side chain of cysteine might not be able to undergo the necessary nucleophilic attack. It further implies that the cysteine found at the DNA binding domain of 
$\mathrm{NF}-\mathrm{KB}$ is less nucleophilic compared to the cysteine residue of IKK since a stronger nucleophile is needed to overcome the inertness of ent-kaurenoic acid. Furthermore, the very hydrophobic nature of ent-kaurenoic acid as well the different potential density behaviour explain the underlying selectivity towards its corresponding target since ent-kaurenoic acid probably adopts a different spatial orientation in order to satisfy the hydrophobic and electrostatic matching with the binding site of the target.

\section{References}

1. Newman D J and Cragg G M, J Nat Prod., 2000, 70, 461-477

2. Newman D, Cragg G and Snader K, Nat Prod Rep., 2000. 17: 215 - 234

3. Costa-Lotufo L V, Cunha G M A, Farias P A M, Viana G S B., Cunha K M A, Pessosa C, Moraes M O, Silviera E R, Gramosa N V and Rao V S N, Toxicon., 2002, 40, $1231-1234$

4. Cunha K M A, Paiva L A F, Santos F A, Gramosa N V, Silviera E R and Rao V S N, Phytother Res., 2003. 17, 320-324

5. Velikova M, Bankova V, Tsetkova I, Kujumgiev A and Marcucci M, Fitoterapia., 2000, 71, 693-696

6. Ragasa C Y, Alimboyoguen A B, Urban S and Raga D D, Nat Prod Comm., 2008, 3(10), 1663- 1666

7. Stumvoll M, Goldstein B and van Haeften T, Lancet., 2005, 365(9467), 1333-1346

8. Karin M and Delhase M, Semin Immunol., 2000, 12(1), 85-98

9. Castrillo A, de las Heras B, Hortelano S, Rodriguez B, Villar A and Bosca L, J Biol Chem., 2001, 276, 15854-15860

10. Giang P M, Jin H Z, Son P T, Lee J H, Hong Y S and Lee J J, J Nat Prod., 2003, 66, $1217-1220$

11. Lee J H, Koo T H, Hwang B Y and Lee J J, J Biol Chem., 2002, 277, 18411-18420

12. Young D C, Computational Drug Design: A Guide for Computational and Medicinal Chemists. John Wiley \& Sons, Inc, New Jersey, 2009

13. Fersht A R, Shi J, Knill - Jones J, Lowe D M, Wilkinson A J, Blow D M, Brick P, Carter P, Waye M M Y and Winter G, Nature, 1985,.314, 235-238

14. Lipinski C, Lombardo F, Dominy B and Feeney P, Adv Drug Delivery Rev., 2001, 46, 3-26

15. Patrick G L, An Introduction to Medicinal Chemistry. Oxford University Press lnc, New York, 1995

16. Palm K, Luthamn K, Ungell A L, Strandlund G, Beigi F, Lundahl P and Artursson P, J Med Chem., 1998, 41, 5382-5392

17. Kelder J, Grootenhuis P, Bayada D, Delbressine L and Ploemen, J Pharm Res., 1999, 16, 1514-1519

18. Naray-Szabo G, J Mol Recognit., 1993, 6, 205-210

19. Bhattacharjee A K, Gupta R K, Ma D and Karle J M, J Mol Recognit., 2000, 13(4), 213-220

20. Pearson R G, Acc Chem Res., 1993. 26, 250 - 255

21. Campos J, del Carmen Nunez M, Rodriguez V, Entrena A, Hernandez-Alcoceba R, Fernandez F, Lacal J C, Gallo M A and Espinosa, Eur J Med Chem., 2001, 36, 215-225

22. Nam W, Ryu J Y, Kim I and Kim C, Tetrahedron Lett., 2002, 43, 5487-5490. 


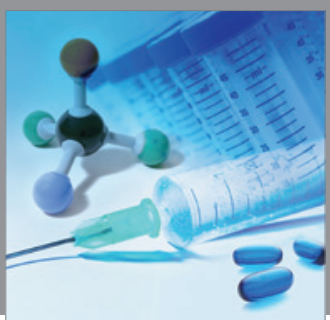

International Journal of

Medicinal Chemistry

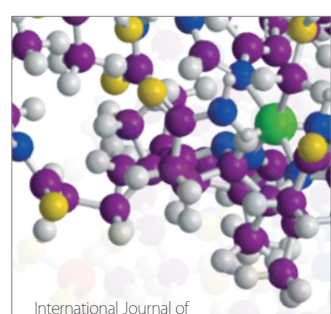

Carbohydrate Chemistry

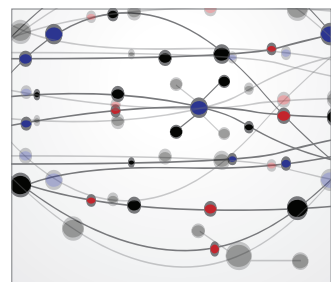

The Scientific World Journal
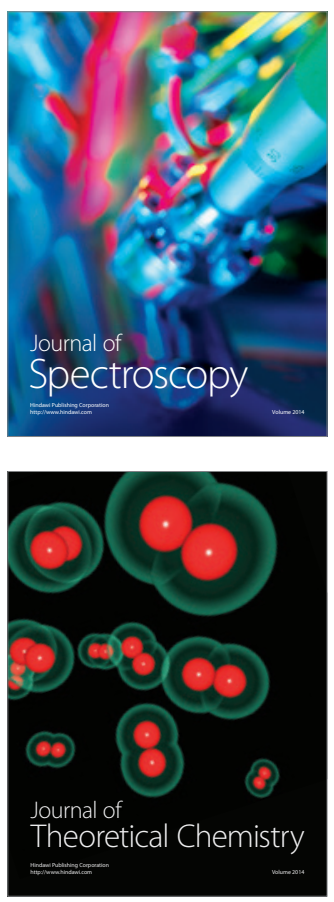
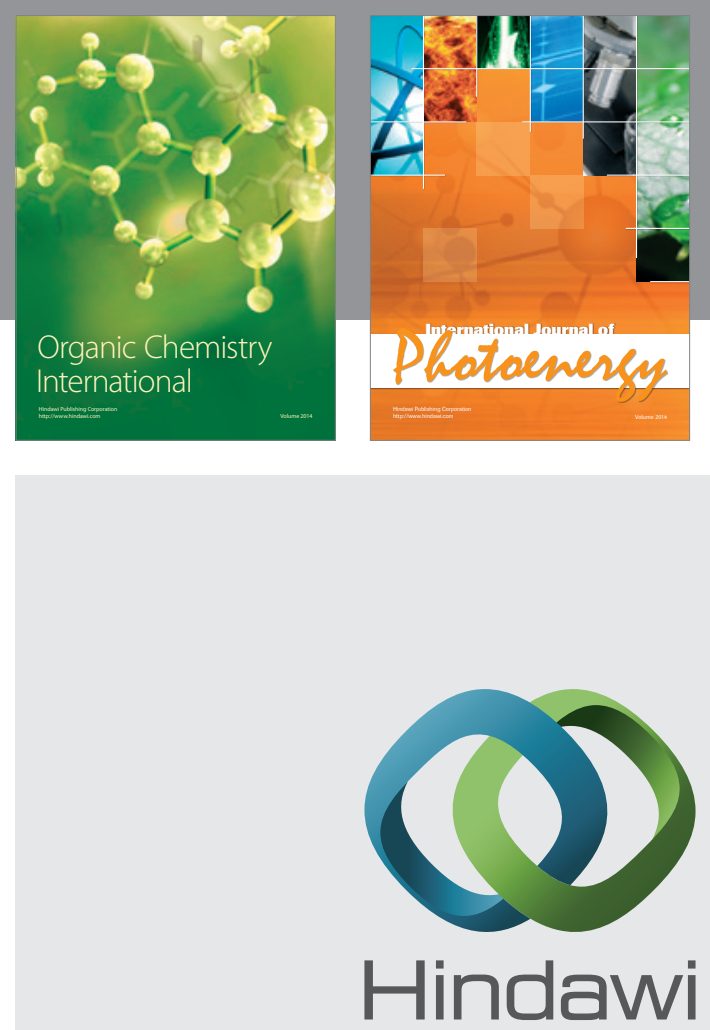

Submit your manuscripts at

http://www.hindawi.com
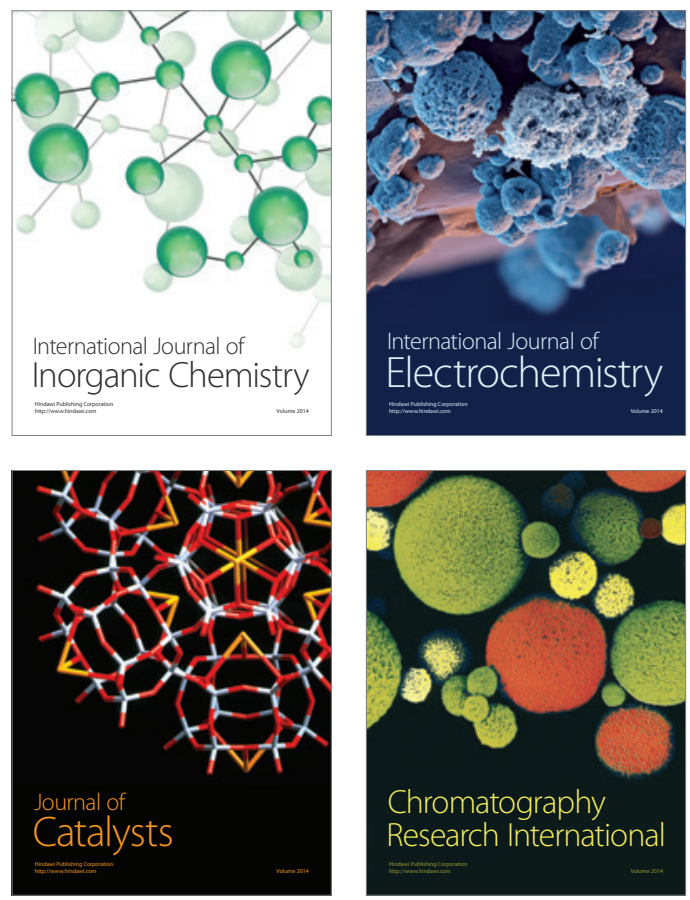
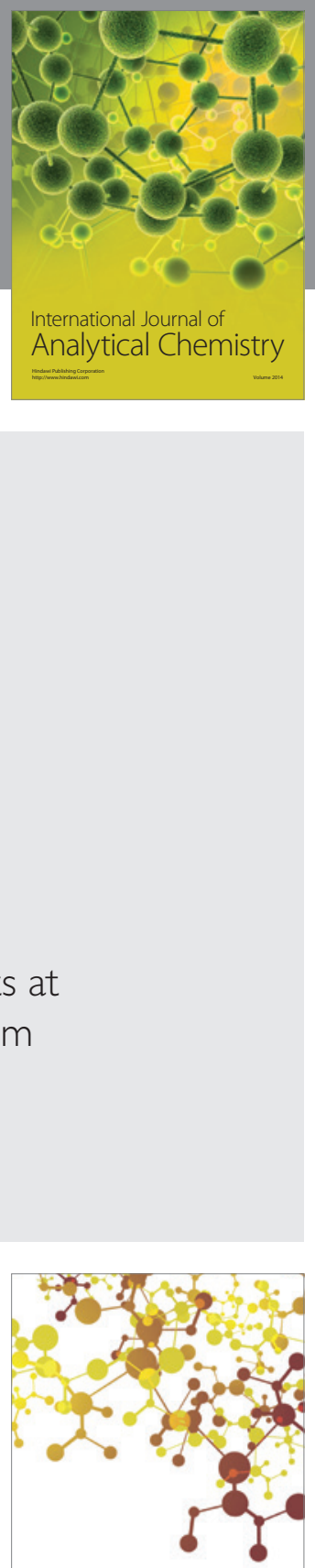

Journal of

Applied Chemistry
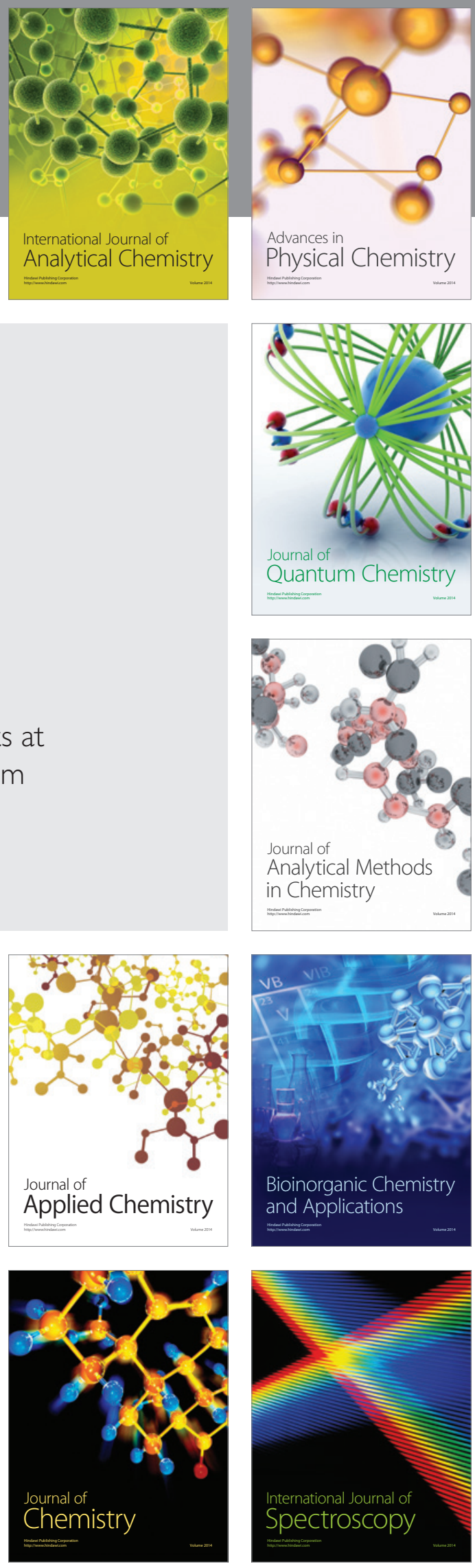